\title{
Short Stay Thyroid Surgery: Can We Replicate the Same in Low Resource Setting?
}

\author{
Naval Bansal, ${ }^{1}$ Sanjay Kumar Yadav, ${ }^{2}$ Saroj Kanta Mishra $(D)$, \\ Kamal Kishore, ${ }^{4}$ Anjali Mishra, ${ }^{3}$ Gyan Chand $\odot{ }^{5},{ }^{5}$ Gaurav Agarwal, ${ }^{3}$ \\ Amit Agarwal, ${ }^{3}$ and Ashok Kumar Verma ${ }^{3}$ \\ ${ }^{1}$ Consultant Breast and Endocrine Surgeon, Fortis Healthcare, Mohali, India \\ ${ }^{2}$ Senior Resident, Department of Endocrine Surgery, Sanjay Gandhi Postgraduate Institute of Medical Sciences, \\ Raebareli Road, Lucknow 226 014, India \\ ${ }^{3}$ Professor, Department of Endocrine Surgery, Sanjay Gandhi Postgraduate Institute of Medical Sciences, Raebareli Road, \\ Lucknow 226 014, India \\ ${ }^{4}$ Provisional Fellow, Department of Anaesthesia and Perioperative Medicine, Royal Hobart Hospital, Hobart, Tasmania 7001, Australia \\ ${ }^{5}$ Additional Professor, Department of Endocrine Surgery, Sanjay Gandhi Postgraduate Institute of Medical Sciences, \\ Raebareli Road, Lucknow 226 014, India
}

Correspondence should be addressed to Saroj Kanta Mishra; skmishra_1956@yahoo.com

Received 15 May 2018; Accepted 25 June 2018; Published 5 August 2018

Academic Editor: Brendan C. Stack Jr.

Copyright (C) 2018 Naval Bansal et al. This is an open access article distributed under the Creative Commons Attribution License, which permits unrestricted use, distribution, and reproduction in any medium, provided the original work is properly cited.

\begin{abstract}
Introduction. The concept of short stay thyroidectomy has been tested and in practice in the developed world; the same has not been replicated in countries with limited resources due to lack of organized healthcare system. So, in this study, we tried to analyze if short stay thyroid surgery can be performed in a cost-effective way in developing countries and also if the endocrine surgical trainee can deliver these services safely. Methods. The study was conducted prospectively from January 2013 to July 2014, at Department of Endocrine Surgery, SGPGIMS, Lucknow, India. Study group included patients undergoing short stay hemithyroidectomy whereas matched patients who qualified for inclusion criteria but did not undergo short stay surgery due to various reasons constituted control group. Outcome in both the groups was compared in terms of complication rates, cost benefit, and patient satisfaction. Subgroup analysis was also done for trainee versus consultant performed short stay thyroid surgery. Results. A total of 439 patients with surgical thyroid disorders were evaluated at our institute during the study period and out of these 110 patients (58 cases and 52 controls) fulfilled the inclusion criteria. Younger patients with low socioeconomic status who were paying out of pocket were found to be more inclined to short stay thyroid surgery. There was no significant difference between the two groups in terms of postanesthetic discharge score (PADS), complication rates, and patients satisfaction; however there was significant reduction $(\mathrm{p}<0.001)$ in hospital cost in short stay group. In subgroup analysis, procedure time was more in trainee performed surgeries; however there was no significant difference in terms of mean PADS and complication rates. Conclusion. Short stay thyroidectomy can provide a better cost-effective alternative to conventional thyroidectomy in patients undergoing thyroid surgery and can be safely performed by endocrine surgical trainees even in a low resource setting.
\end{abstract}

\section{Introduction}

In today's era with the advancement of technology and safe surgical techniques, "day care surgery" (admission, surgery, observation, and discharge on the same working day) and "short stay surgery" (surgery followed by overnight observation with discharge the following morning; typical stay duration of $23 \mathrm{~h}$ ) are being undertaken for a growing list of surgical procedures including thyroid surgery $[1,2]$. Though the concept has been tested and is in practice in the developed countries, the same has not been replicated in countries with limited resources $[3,4]$. Day care/short stay thyroid surgery has been found to be as safe as in-patient thyroidectomy and also has added advantages in terms of 
TABLE 1: Inclusion and exclusion criteria [9].

\begin{tabular}{ll}
\hline Inclusion criteria & Exclusion criteria \\
\hline Clinical & (i) Previous thyroid surgery \\
\hline (i) Patients of all age group & (ii) Ultrasound-estimated thyroid volumes $>80 \mathrm{ml}$ \\
(ii) Patients of ASA grades I \& II & (iii) Patients on drugs that have an effect on coagulation \\
(iii) First neck surgery & \\
(iv) Euthyroidism & \\
(v) Ultrasound estimated volume $<80 \mathrm{ml}$ & \\
(vi) Patients undergoing hemithyroidectomy & \\
Social-logistic & \\
\hline (i) Established autonomy at admission & \\
(ii) Adequate home support at discharge & \\
(iii) Able to communicate &
\end{tabular}

reduced risk of infection, shorter convalescence time, high patient satisfaction, and low cost [5-7]. However, the majority of developing nations still lack adequate resources to practice day care/short stay thyroidectomy safely. Further, poor financial status of patients and lack of universal health insurance policy force patients to bear the expense of surgical care out of pocket and shortage of skilled surgeons and anesthesiologists adds to the gravity of the situation. While the economic and social satisfaction outcomes have been investigated in great length, competency of surgeons to practice the procedure has not yet been looked into [8]. So, in this study, we aim to analyze if short stay thyroid surgery can be replicated in low resource setting in a cost-effective way and also if the endocrine surgical trainees can deliver these services safely.

\section{Methods}

The study was conducted prospectively from January 2013 to July 2014, in the Department of Endocrine Surgery, Sanjay Gandhi Postgraduate Institute of Medical Sciences, Lucknow, India, a tertiary care academic institute. Study group included patients undergoing short stay thyroidectomy and control group included matched patients who qualified for inclusion criteria but did not undergo short stay surgery due to various reasons (refusal to consent for short stay surgery or sociologistic problems). Outcome in both groups was compared in terms of complication rates, cost benefit, and patient satisfaction. Subgroup analysis was also done for trainee versus consultant performed short stay thyroid surgery. Short stay was defined as surgery followed by overnight observation with discharge on the following morning; total hospital stay duration is less than 23 hours. Inclusion and exclusion criteria for the study group are listed in Table 1. Surgical procedures were performed under general anaesthesia with endotracheal intubation. Patients were injected with intravenous Midazolam, Fentanyl, and Propofol and were maintained on Vecuronium and Desflurane. Antiemetic in the form of Ondansetron was given in all patients before shifting the patient from operating room to the postoperative observation ward. All patients received antibiotic prophylaxis and for pain single dose of intravenous Paracetamol and thereafter oral ibuprofen for three days was prescribed. Thyroidectomy was conducted through cervicotomy approach in a standard way. Oral diet was allowed, as tolerated, during the immediate postoperative period. All patients were evaluated next day morning according to the Postanesthetic Discharge Scoring System (PADSS) [10] and were discharged if score was more than nine. On discharge, each patient was counseled and received written and verbal instructions for wound management, postoperative pain, and signs/symptoms associated with possible complications. Patients were followed up at two weeks, six weeks, and at six months following surgery to look for short and long term complications. Results were analyzed using SPSS version 17.0. Independent $t$ test and Chi-square tests were used for statistical analysis.

\section{Results}

During the study period a total of 439 patients with surgical thyroid disorders were evaluated at our institute and 110 patients fulfilled the inclusion criteria. Out of these, 58 $(52.73 \%)$ patients consented for short stay thyroid surgery and were included in study group whereas the remaining 52 (47.23\%) were recruited in control group. Detailed clinical and socioeconomic profile is listed in Table 2.

There was significant difference in acceptance rate among the younger patients with low socioeconomic status. Also patients who were paying hospital bills out of pocket opted more for short stay thyroid surgery ( $p$ 0.036). However, there was no statistical significant difference between two groups in terms of mean distance from hospital, ASA grade, and thyroid volume.

There was no significant difference among both groups in terms of mean postanesthetic discharge score and complication rates (Table 3 ). In short stay group, one patient developed noncompressive hematoma within six hours of surgery which was managed conservatively and one had urinary retention in postoperative period. One postmenopausal female patient developed symptomatic hypocalcaemia and required calcium and vitamin D supplements. All three patients (5.17\%) required extended stay for 48-72 hours. There was significant difference in terms of hospital stay and cost of treatment among both the groups; however, there was no significant difference in terms of patient's satisfaction. None of the 
TABLE 2: Patients clinical and socioeconomic profile.

\begin{tabular}{|c|c|c|c|}
\hline Items & Study group $(\mathrm{n}=58)$ & Control group $(n=52)$ & $\mathrm{p}$ value \\
\hline Mean Age (in years) & $31.66 \pm 10.15$ & $37.60 \pm 13.51$ & 0.010 \\
\hline Sex $(M: F)$ & $1: 4.3$ & $1: 2.3$ & 0.151 \\
\hline \multicolumn{4}{|l|}{ Financial status* } \\
\hline (i) Low income & $3(5.17 \%)$ & 0 & 0.001 \\
\hline (ii) Middle income & $49(84.48 \%)$ & $31(59.62 \%)$ & \\
\hline (iii) High income & $6(10.34 \%)$ & $21(40.38 \%)$ & \\
\hline \multicolumn{4}{|l|}{ Mode of payment } \\
\hline (i) Self & $53(91.38 \%)$ & $40(76.92 \%)$ & 0.036 \\
\hline (ii) Insurance & $5(8.72 \%)$ & $12(23.08 \%)$ & \\
\hline Mean Distance (in Km) & $210.59 \pm 157.52$ & $231.58 \pm 201.04$ & 0.541 \\
\hline Thyroid volume (in ml) & $23.79 \pm 17.69$ & $26.02 \pm 18.92$ & 0.528 \\
\hline \multicolumn{4}{|l|}{ ASA grade } \\
\hline I & $47(81.03 \%)$ & $40(76.92 \%)$ & 0.597 \\
\hline II & $11(18.97)$ & $12(23.08 \%)$ & \\
\hline
\end{tabular}

* Based on OECD working paper (http://www.oecd.org/dev/44457738.pdf).

TABLE 3: Outcome analysis.

\begin{tabular}{lcc}
\hline Items & Study group $(\mathrm{n}=58)$ & Control group (n=52) \\
\hline Mean PADS & 9.79 & 9.71 \\
Complications & & 0.325 \\
(i) Hemorrhage & $1(1.72 \%)$ & $1(1.92 \%)$ \\
(ii) Hoarseness of voice & & $2(3.85 \%)$ \\
$\quad$ (a) Transient & $1(1.72 \%)$ & 0 \\
(b) Permanent & 0 & $4(7.69 \%)$ \\
(iii) Seroma/ wound infection & $6(10.34 \%)$ & 0 \\
(iv) Others & $2(3.45 \%)$ & $106.44 \pm 39.74$ \\
Total duration of hospital stay (in hours) & $25.05 \pm 9.5$ & $14041 \pm 4220$ \\
Cost of treatment (INR) & $10409 \pm 1355$ & 0.513 \\
Satisfaction level based on duration of hospital stay & & $42(80.77 \%)$ \\
(i) Appropriate & $47(81.03 \%)$ & $10(19.23 \%)$ \\
(ii) Should have remained longer & $11(18.97 \%)$ & $<0.001$ \\
Final Histopathology & & 0.001 \\
(i) Benign & $52(89.65 \%)$ & 0.972 \\
(ii) Malignant & $6(10.35 \%)$ & $7(13.46 \%)$ \\
\hline
\end{tabular}

patients required readmission. Eleven (18.97\%) patients felt that they should have been kept for a longer duration (pain: 5, apprehension: 3 , and seroma: 3 patients).

Among 58 short stay thyroid surgeries, endocrine surgery trainees performed 39 hemithyroidectomies whereas consultants performed 19 hemithyroidectomies. There was no significant difference in terms of mean thyroid volume in both the groups (Table 4 ). Procedure time was more in trainee performed surgeries $(124.89 \pm 24.64$ versus $108.77 \pm 30.79$, p 0.051 ); however, there was no difference in terms of mean postanesthetic discharge score and complication rates in both the groups. In trainee performed subgroup, one patient had noncompressive hematoma and one developed hypocalcaemia.

\section{Discussion}

To the best of author's knowledge this study is unique in addressing safety, economics, and competency aspect of short stay thyroid surgery in a low resource scenario. As per the World Bank report, India belongs to lower middle income country group with $24.7 \%$ population living in extreme poverty $[11,12]$. So, short stay surgeries can provide a more realistic approach for cost-effective healthcare if it can be achieved with reasonable outcome. In our study we found that patients who belong to low and middle income group were more inclined for short stay thyroidectomy. Similarly, short stay thyroidectomy was more often opted by patients paying hospital expenses out of pocket, so this subset of 
TABLE 4: Trainee versus consultant performed short stay thyroidectomy.

\begin{tabular}{|c|c|c|c|}
\hline Items & Trainee performed $(n=39)$ & Consultant performed $(\mathrm{n}=19)$ & $\mathrm{p}$ value \\
\hline Median thyroid volume (in $\mathrm{ml}$ ) & $25.66 \pm 18.48$ & $20.05 \pm 15.79$ & 0.263 \\
\hline Procedure time (in minutes) & $124.89 \pm 24.64$ & $108.77 \pm 30.79$ & 0.051 \\
\hline PADS & $9.79 \pm 0.409$ & $9.79 \pm 0.419$ & 0.963 \\
\hline \multicolumn{4}{|l|}{ Complications } \\
\hline (i) Hemorrhage & $1(2.56 \%)$ & 0 & 0.686 \\
\hline \multicolumn{4}{|l|}{ (ii) Hoarseness of voice } \\
\hline (a) Transient & 0 & $1(5.26 \%)$ & \\
\hline (b) Permanent & 0 & 0 & \\
\hline (iii) Seroma / wound infection & $3(7.69 \%)$ & $3(15.79 \%)$ & \\
\hline (iv) Others & $2(5.13 \%)$ & 0 & \\
\hline \multicolumn{4}{|l|}{ Final Histopathology } \\
\hline (i) Benign & $35(89.74 \%)$ & $17(89.47 \%)$ & 0.975 \\
\hline (ii) Malignant & $4(10.26 \%)$ & $2(10.53 \%)$ & \\
\hline
\end{tabular}

patients should be the initial target population for offering short stay surgery in developing countries.

Short stay thyroidectomy appears to be more feasible than day care thyroidectomy in a developing country like India as patients travel a longer distance due to availability of only a few specialized centers and lack of primary care physician unlike developed countries. Contrary to American Thyroid Association (ATA) guideline on outpatient thyroidectomy, in our study mean distance was $210.59 \pm 157.52 \mathrm{Km}$, signifying that distance should not be kept as criterion in developing countries if we are offering short stay services as compared to outpatient thyroidectomy [8]. Postoperative hemorrhage is the single biggest concern in outpatient thyroid surgery with incidence of 0.9 to $2.1 \%$ [11]. One of our patients (1.73\%) had noncompressive hematoma within six hours of surgery and was managed conservatively and did not require reoperation. More than $50 \%$ postoperative hemorrhages occur after six hours of surgery and studies have shown that postthyroidectomy bleed related mortality could be prevented by keeping the patients under observation for a little longer period [13, 14]. Hence, we feel that short stay in contrast to day care would be preferable in low resource scenario. One patient had temporary hoarseness of voice which recovered within three months without any intervention. Six patients developed seroma; out of these, three patients required aspirations. Both groups had similar level of satisfaction in terms of length of hospital stay; however pain and apprehension were two major factors decreasing the satisfaction level among study group. Proper counseling and other modalities of pain relief like cervical plexus block could have improved the satisfaction level [15].

Systemic studies have been conducted in various surgical specialties and they have compared the outcome of trainee performed surgeries; in terms of increased operating time, increased reoperation and readmissions rates [16-18] and similarly complication rates of thyroidectomy are more if performed by unskilled surgeons or surgeons with little experience [19-21]. Studies have shown that longer procedure time is associated with increased perioperative morbidity $[6,22]$. In our study trainees took more time for hemithyroidectomy; however there was no difference in terms of postanesthetic discharge score and wound related morbidity. One patient in study group had acute urinary retention and one had dilutional hypocalcaemia that could be attributed to longer procedure time; however it was statistically nonsignificant.

Internationally, an income of less than $\$ 1.25$ per day per head of purchasing power parity is defined as extreme poverty and by this estimate about $24.7 \%$ of Indians live in extreme poverty $[10,11]$. Average cost of in-patient hemithyroidectomy in matched sample at our hospital is $\$ 220$; so an average sum of $\$ 60$ (more than monthly income of $24.7 \%$ population) was saved per patient while maintaining safety and efficacy of surgical procedure, proving cost effectiveness of this approach.

This study has reported experience from a developing world. We preferred to restrict ourselves only to hemithyroidectomy in contrast to the other thyroid surgical procedures performed on short stay basis in developed countries in view of limited resources in public healthcare to attend to emergencies and thus compromising safety of the patients. However, with large experience, improved community healthcare support, and adoption of telehomecare, other thyroid surgical procedures can be considered for short stay surgery in due course of time.

\section{Conclusion}

Short stay thyroidectomy can provide a better cost-effective alternative to conventional in-patient thyroid surgery and can be safely performed by endocrine surgery trainees even in a low resource setting.

\section{Data Availability}

The data used to support the findings of this study are available from the corresponding author upon request.

\section{Conflicts of Interest}

The authors declare that they have no conflicts of interest. 


\section{References}

[1] R. Mirnezami, A. Sahai, A. Symes, and T. Jeddy, "Day-case and short-stay surgery: The future for thyroidectomy?" International Journal of Clinical Practice, vol. 61, no. 7, pp. 1216-1222, 2007.

[2] G. H. Sun, S. Demonner, and M. M. Davis, "Epidemiological and economic trends in inpatient and outpatient thyroidectomy in the United States, 1996-2006," Thyroid, vol. 23, no. 6, pp. 727733, 2013

[3] K. L. Yerzingatsian, "Short-stay thyroidectomy - Trends in length of post-operative hospitalisation over a period of 10 years in a developing country central hospital," South African Journal of Surgery, vol. 40, no. 2, 81 pages, 2002.

[4] R. A. Dedivitis, E. G. Pfuetzenreiter, M. A. Castro et al., "Analysis of safety of short-stay thyroid surgery," Acta Otorhinolaryngologica Italica, vol. 29, no. 6, pp. 326-330, 2009.

[5] S. K. Snyder, K. S. Hamid, C. R. Roberson et al., "Outpatient thyroidectomy is safe and reasonable: experience with more than 1,000 planned outpatient procedures," Journal of the American College of Surgeons, vol. 210, no. 5, pp. 575-582, 2010.

[6] S. I. Sahmkow, N. Audet, S. Nadeau, M. Camireá, and D. Beaudoin, "Outpatient thyroidectomy: Safety and patients' satisfaction," Journal of Otolaryngology - Head and Neck Surgery, vol. 41, no. 1, pp. S1-S12, 2012.

[7] C. R. McHenry, "“Same-day" thyroid surgery: an analysis of safety, cost savings, and outcome," The American Surgeon, vol. 63, pp. 586-589, 1997.

[8] D. J. Terris, S. Snyder, D. Carneiro-Pla et al., "American thyroid association statement on outpatient thyroidectomy," Thyroid, vol. 23, no. 10, pp. 1193-1202, 2013.

[9] G. Dionigi, F. Rovera, G. Carrafiello, L. Boni, and R. Dionigi, "Ambulatory thyroid surgery: Need for stricter patient selection criteria," International Journal of Surgery, vol. 6, no. 1, pp. S19S21, 2008.

[10] F. Chung, V. W. S. Chan, and D. Ong, "A Post-Anesthetic Discharge Scoring System for home readiness after ambulatory surgery," Journal of Clinical Anesthesia, vol. 7, no. 6, pp. 500-506, 1995.

[11] http://data.worldbank.org/about/country-and-lending-groups.

[12] http://povertydata.worldbank.org/poverty/country/IND.

[13] P. Leyre, T. Desurmont, L. Lacoste et al., "Does the risk of compressive hematoma after thyroidectomy authorize 1-day surgery?" Langenbeck's Archives of Surgery, vol. 393, no. 5, pp. 733-737, 2008.

[14] A. E. Schwartz, O. H. Clark, P. Ituarte, and P. Lo Gerfo, “Thyroid surgery-the choice," The Journal of Clinical Endocrinology and Metabolism, vol. 83, pp. 1097-1105, 1998.

[15] R. J. Egan, J. C. Hopkins, A. J. Beamish, R. Shah, A. G. Edwards, and J. D. T. Morgan, "Randomized clinical trial of intraoperative superficial cervical plexus block versus incisional local anaesthesia in thyroid and parathyroid surgery," British Journal of Surgery, vol. 100, no. 13, pp. 1732-1738, 2013.

[16] S. Q. Kern, M. B. Lustik, L. P. Mcmann, G. P. Thibault, and J. R. Sterbis, "Comparison of outcomes after minimally invasive versus open partial nephrectomy with respect to trainee involvement utilizing the American College of Surgeons national surgical quality improvement program," Journal of Endourology, vol. 28, no. 1, pp. 40-47, 2014.

[17] E. Igwe, E. Hernandez, S. Rose, and S. Uppal, "Resident participation in laparoscopic hysterectomy: Impact of trainee involvement on operative times and surgical outcomes," American
Journal of Obstetrics \& Gynecology, vol. 211, no. 5, pp. 484484.e7, 2014.

[18] S. W. Ross, B. Oommen, M. Kim et al., "A little slower, but just as good: postgraduate year resident versus attending outcomes in laparoscopic ventral hernia repair," Surgical Endoscopy, vol. 28, no. 11, pp. 3092-3100, 2014.

[19] A. Hauch, Z. Al-Qurayshi, P. Friedlander, and E. Kandil, "Association of socioeconomic status, race, and ethnicity with outcomes of patients undergoing thyroid surgery," JAMA Otolaryngology - Head and Neck Surgery, vol. 140, no. 12, pp. 1173-1183, 2014.

[20] E. Kandil, S. I. Noureldine, A. Abbas, and R. P. Tufano, "The impact of surgical volume on patient outcomes following thyroid surgery," Surgery, vol. 154, no. 6, pp. 1346-1353, 2013.

[21] J. A. Sosa, H. M. Bowman, J. M. Tielsch, N. R. Powe, T. A. Gordon, and R. Udelsman, "The importance of surgeon experience for clinical and economic outcomes from thyroidectomy," Annals of Surgery, vol. 228, no. 3, pp. 320-330, 1998.

[22] T. D. Jackson, J. J. Wannares, R. T. Lancaster, D. W. Rattner, and M. M. Hutter, "Does speed matter? the impact of operative time on outcome in laparoscopic surgery," Surgical Endoscopy, vol. 25, no. 7, pp. 2288-2295, 2011. 


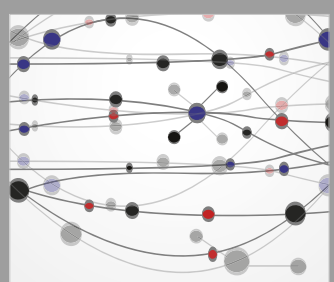

The Scientific World Journal
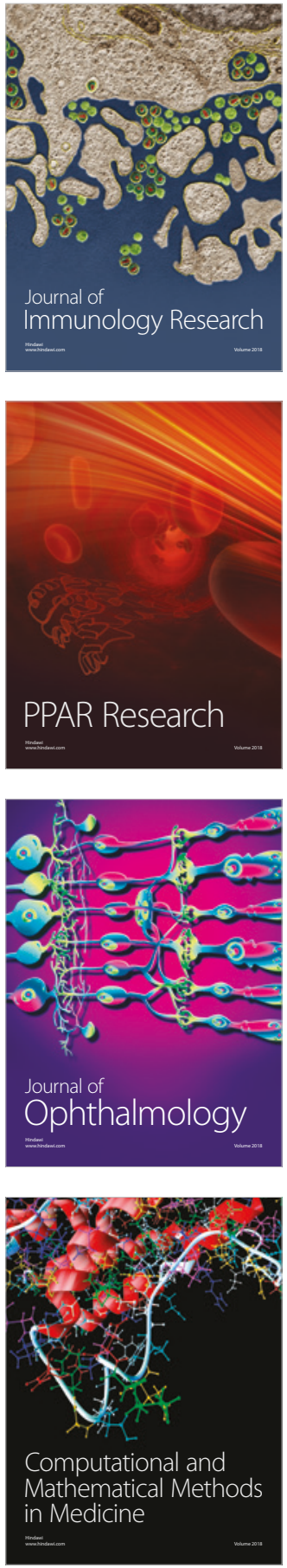

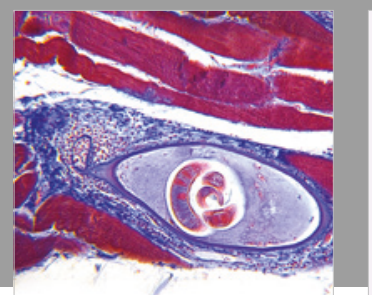

Gastroenterology Research and Practice

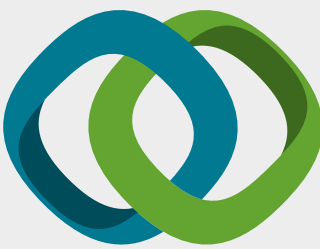

\section{Hindawi}

Submit your manuscripts at

www.hindawi.com
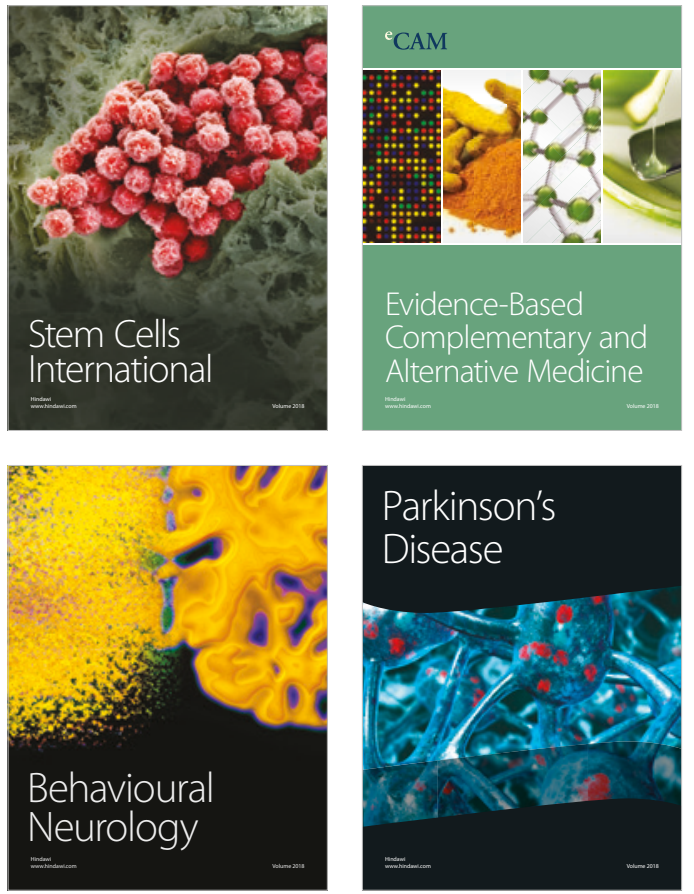

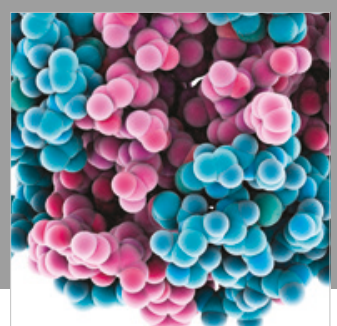

ournal of

Diabetes Research

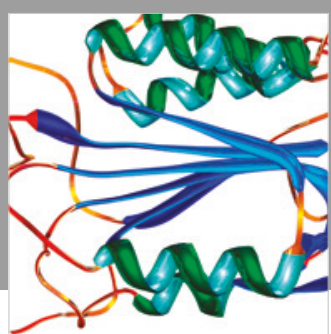

Disease Markers
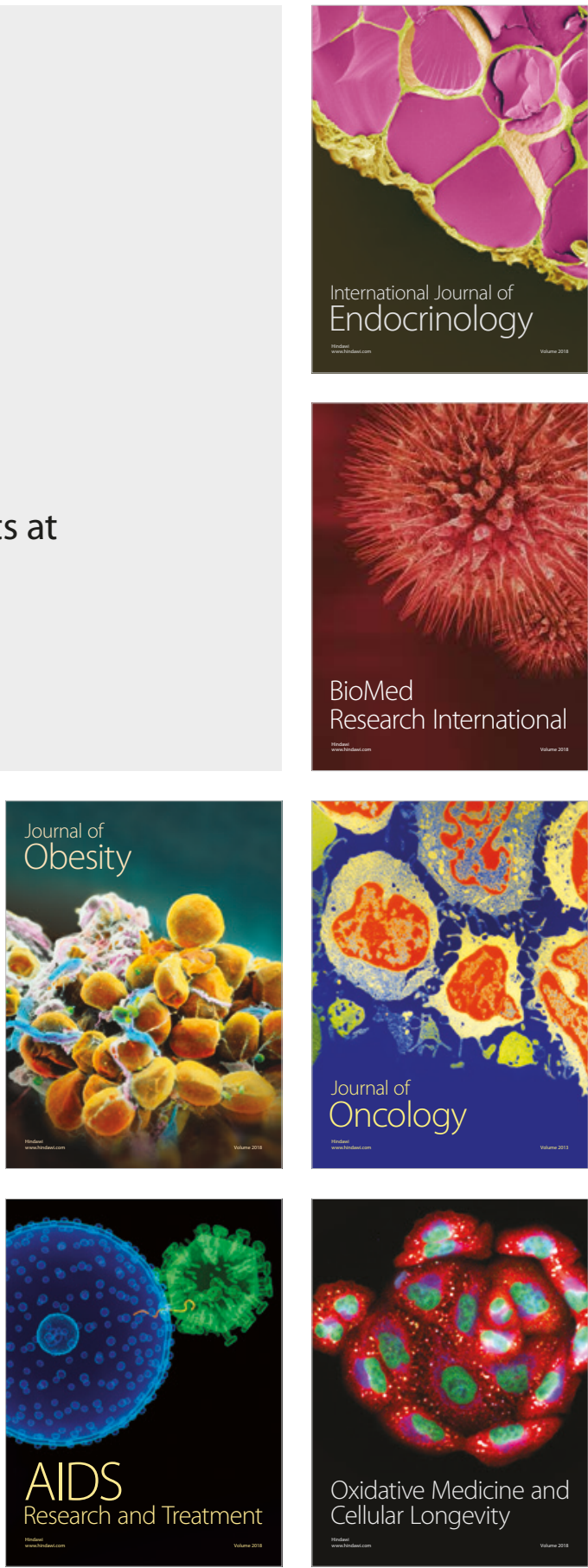